\title{
Neonatal cornea opacity
}

\section{Opinion}

Rarely are advances in medical therapy, either drug or surgical technique related, quickly adopted and disseminated. Some developments are controversial by their very nature, while others are inherently difficult to deploy. Physicians guided by the concept of "Do No Harm" will often elect for a consensus to develop prior to incorporating new developments into their practice. Oversight and regulatory precepts cannot be ignored and in the United States delayed Food and Drug Administration approvals as well as the absence of reimbursement by third party payers are often controlling factors. In the case of rare disease entities many years may be required for the collection of a sufficient number of cases prior to the reporting of long term results necessary to evaluate the true efficacy of new procedures. Peters Anomaly with its associated dysgenesis is associated with aberrations occurring in the first trimester of pregnancy due to genetic events which disrupt anterior segment formation and the cleavage of the anterior chamber of the eye.

The first reported case of the implantation of the Boston type I keratoprosthesis in infant's dates to 2003, and as yet there have been no long term reports. While such a reports are in preparation, investigation into the factors responsible reveals the root cause of delay to be the rarity of the disease coupled with the small number of infants treated, as well as the limited number of institutions willing to participate. Further analysis has uncovered a number of logistical difficulties which combine to render difficult the performance of the newly advocated surgical procedure. Often the diagnosis of cornea blindness is made by the mother a few days following the birth of the child when the absence of normal appearing ocular anatomy becomes apparent. Invariably numerous consultations and examinations ensue, prior making the difficult decision to seek the new procedure, in the face of contrary advice. The severity of the condition not infrequently results in the opinion that nothing can be done.

Upon receipt of a referral by the receiving institution, the complicated process of collecting results of previous evaluations, and interventions begins. This administrative process is both labor and time intensive. If irreversible amblyopia is to be avoided images must be presented to the retina during the early months of life. Once all records have been reviewed an actual examination must be conducted not by one but often three ophthalmic subspecialists. The receiving institution is usually at a geographical location far removed from the child's home. Travel, visas, and authorizations must be obtained. The receiving institution must arrange for a series of evaluations, many conducted under anesthesia, as well as specific requirements for tissue and device to be implanted. Logging in distant cities or countries must be available and if surgery is to proceed the subspecialists involved must all be available. Special post-operative medications and follow-
Volume 5 Issue I - 2016

\section{Catherine EAquavella}

Department of Ophthalmology, University of Rochester Flaum Eye Institute, USA

Correspondence: Catherine E Aquavella, Distinguished Professor of Ophthalmology, University of Rochester Flaum Eye Institute, 60I Elmwood Avenue, Box 659 Rochester, NY I4642, USA,Email james_aquavella@urmc.rochester.edu

Received: September 28, 2016 | Published: September 28, 2016

up examinations must be scheduled. Reports need to be prepared for referring specialists. And ultimately facile communication between distant physicians must be established on a routine basis so that all physicians can be appraised of the results of examinations conducted locally, and compliance with recommended protocols is assured.

This process is difficult and associated with a financial burden for both family and receiving institution since large amounts both human and physical resources are necessary in an attempt to provide the infant an opportunity for some useful sight. The surgical team involves pediatric, glaucoma, cornea, and vitreoretinal ophthalmic sub specialists. Surgical nurses and technicians, clinical ophthalmic technicians, NICU nurses, pediatric anesthesiologists, surgical schedulers, and administrative secretaries are essential members of the support team. These varied duties must be coordinated. A provision must be in place to handle unscheduled visits or complications. While it is clear that ultimate results will vary with the intensity of the disease process and not all infants can be provided with an opportunity for sight, early reports indicate that some may be afforded an opportunity where none existed prior to the development of this procedure. We await the long term report of results achieved, as well as the involvement of a greater number of ophthalmic surgeons and their institutions.

\section{Acknowledgments}

None.

\section{Funding}

None.

\section{Conflicts of interest}

The authors declare there is no conflict of interests. 